\title{
Kawasaki Disease
}

\author{
Hirohisa Kato
}

Background and History . . . . . . . . . . . . . . . . 979

Epidemiology . . . . . . . . . . . . . . . . . . . . . 980

Pathology . . . . . . . . . . . . . . . . . . . . . . 980

Pathogenesis and Etiology ................ 980

Clinical Presentations ................. 981

\section{Key Points}

- Kawasaki disease is an acute systemic vasculitis syndrome in infants and young children of unknown etiology, which mainly affects small and medium-sized arteries, particularly the coronary artery.

- Clinical features include an acute febrile illness associated with mucocutaneous involvements and lymphadenopathy.

- The coronary aneurysms develop in about $10 \%$ to $20 \%$ of patients, some of which may cause myocardial infarction or lead to ischemic heart disease.

- Combinations of high-dose gamma globulin and aspirin are the essential treatments for acute Kawasaki disease patients.

- Bypass surgery and the catheter intervention are the optional treatments for the coronary artery sequelae of Kawasaki disease.

- Some patients with coronary artery sequelae may develop into adult coronary artery disease.

Kawasaki disease (KD) is an acute vasculitis syndrome in infants and young children of unknown etiology, which mainly affects small and medium-sized arteries, particularly the coronary artery. The clinical feature is an acute febrile illness associated with mucocutaneous involvement and lymphadenopathy. Coronary aneurysms develop in about $10 \%$ to $20 \%$ of patients, some of which may cause myocardial infarction or ischemic heart disease in children and young adults. High-dose gamma globulin and aspirin are the essential treatments for acute Kawasaki disease in patients.

\section{Background and History}

Kawasaki disease (KD) or mucocutaneous lymph node syndrome (MCLS) is an acute arteritis of small and mediumsized arteries, particularly coronary arteries. ${ }^{1}$ The cardiovascular problems in $\mathrm{KD}$ include coronary artery
Diagnostic Techniques ................... 983

Evidence-Based Treatment and Management . . . . . . . 985 Prognosis and Long-Term Issues . . . . . . . . . . . . . . 989 Guidelines ............................ 992 Summary . . . . . . . . . . . . . . . . . . 992

lesions that develop aneurysm formation, thrombotic occlusion, progression to coronary artery disease, and premature atherosclerosis. ${ }^{2}$ These problems may cause myocardial infarction or sudden death in the young. The disease was first described by Dr. Tomisaku Kawasaki ${ }^{3}$ in Japan, and he recognized the first case in 1961. At that time, this disease was thought to be benign and the prognosis good because all acute symptoms disappeared within 2 or 3 weeks, and the patient was usually doing well. Yamamoto and Kimura ${ }^{4}$ reported a case complicated with myocarditis; however, the presence of coronary artery involvement was not identified. In 1970, the first nationwide survey in Japan showed that $1.7 \%$ of the patients died from acute myocardial infarction; autopsies showed coronary arteritis accompanied by aneurysms and thrombotic occlusions. At that time, most pediatricians believed that Kawasaki disease was complicated with vasculitis only in small numbers of fatal cases, because of the great contrast in prognosis between these rare fatal cases and the large number of nonfatal cases that were asymptomatic.

In 1973 , Kato et al..$^{5}$ performed the coronary angiography in 20 infants and children when their acute symptoms had remitted. Twelve of these patients showed multiple coronary aneurysms in both the right and left coronary arteries, which was the first recognition that coronary artery aneurysms are present not only in fatal cases but also in survivors, even if the patient is free from cardiac symptoms. From pathologic studies and the coronary angiographic findings, KD is now recognized to be a systemic arteritis syndrome in small and medium-sized arteries in infants and young children.

In the past, $\mathrm{KD}$ may have been misdiagnosed as another disease, such as scarlet fever, measles, rheumatic fever, juvenile rheumatoid arthritis, Stevens-Johnson syndrome, and infantile polyarteritis, which are now listed in the differential diagnosis of KD. ${ }^{6}$ Landing and Larson $^{7}$ reported that pathologic findings are identical between fatal KD and infantile polyarteritis, which was described only by autopsy cases. It is now believed that KD and infantile polyarteritis are both a systemic arteritis syndrome in infants and young 
children with a wide spectrum of clinical symptoms and disease severity.

\section{Epidemiology}

Epidemiology in Japan where KD is most prevalent has been well documented by the research committee organized by the Health and Welfare Department of the Japanese government. ${ }^{8} \mathrm{~A}$ summary of the epidemiologic picture is as follows: Kawasaki disease has increased in incidence since 1968, and the number of patients affected is now more than 190,000. Kawasaki disease affects over 6000 children each year in Japan, and the incidence is increasing by year to 150 per 100,000 children under 4 years of age. The age affected starts at 1 month of age and peaks at 1 year of age. Fifty percent of cases are under 2 years of age. Cases over 10 years of age are quite rare. Boys are affected more than girls $(1.5: 1)$. It is evident that $\mathrm{KD}$ is more prevalent in the Japanese and in Orientals than in Caucasians. There are no geographical differences in urban or rural areas, and there is wide prevalence throughout all regions of Japan. Seasonal variation is not distinct; however, there are small peaks in winter and spring. The recurrence rate is about $3.3 \%$, and sibling cases are only $1 \%$ to $2 \%$. Time-space clustering or outbreaks in communities are recognized; however, there is no evidence of personto-person transmission. In 1979, 1982, and 1986, there were large outbreaks in Japan, but in the past 10 years, there have not been similar occurrences.

The global epidemiology of KD was described by Nakamura and Yanagawa, ${ }^{9}$ and accordingly, KD is now widely recognized all over the world. However, it is primarily identified in Japan and East Asia. In the United States, the incidence has been estimated from hospital discharge data, which showed that 4248 patients with KD were hospitalized in the year 2000. The incidence is most common in Asian and Pacific Island descendants $(32.5 / 100,000$ children less than 5 years of age), in non-Hispanic African Americans (16.9), and in Hispanics (11.1), and lowest in whites (9.1).

The fatality rate was $2 \%$ in the 1970 s and declined to $0.1 \%$ in the 1990 s in Japan. In the U.S., the in-hospital mortality rate is $0.17 \%$ during which the peak mortality occurs 15 to 45 days after the onset of illness. ${ }^{10}$

\section{Pathology}

Based on Hamashima's analyses of $37 \mathrm{KD}$ autopsy cases, the Japanese Kawasaki Disease Research Committee (1980) reported the following pathomorphologic findings. ${ }^{11,12}$ Kawasaki disease is an acute inflammatory disease with systemic arteritis that is distinguishable from classic periarteritis nodosa of Kussmaul-Maier type, which is a progressive and recurrent arteritis with marked fibrinoid necrosis, but with rare pulmonary vasculitis. Kawasaki disease is an acute, self-limited inflammatory disease with rare and mild fibrinoid necrosis. Infantile polyarteritis resembles KD in many pathologic aspects, ${ }^{7}$ but further studies are needed. Coronary artery aneurysms are usually present at autopsy.

With KD, the arteritis is characterized by an acute inflammation lasting about 7 weeks. The course of arteritis is classified into four stages according to the duration of illness, as follows: Stage 1 ( 1 to 2 weeks of illness): perivasculitis and arteritis of the microvessels, small arteries, and veins; inflammation of intima, externa, and perivascular areas in the midsized and large arteries; edema and inflammation with leukocytes and lymphocytes. Stage 2 (3 to 4 weeks of illness): inflammatory changes of intima, media, externa, and perivascular areas in the midsized arteries with focal panvasculitis; aneurysms with thrombi and stenosis in the midsized arteries, especially in the coronary arteries; edema (exudative stage), infiltration with monocytes or necrosis (infiltrative stage) and cellular granulation with increase of capillaries. Stage 3 (5 to 7 weeks of illness): granulation in the midsized arteries. Stage 4 (more than 7 weeks of illness): scar formation and intimal thickening with aneurysm, thrombi, and stenosis in the midsized arteries /generally no acute inflammation in the vessels). These findings may persist until adulthood.

Myocarditis (interstitial myocarditis with mild necrosis) involves the patient's conduction systems. Myocardial biopsy demonstrated that $80 \%$ of the patients studied had myocarditis, with or without coronary artery lesions. ${ }^{13}$ At autopsy, pericarditis, endocarditis, cholecystitis, cholangitis, pancreatic ductitis, sialoadenitis, meningitis, and lymph adenitis are frequently seen.

\section{Pathogenesis and Etiology}

The etiology of KD remains unknown despite extensive investigations. Based on clinical features and epidemiologic data, KD is now considered to be an infectious etiology of unknown agent(s) or an infection-triggered immune disorder. A variety of possible causative agents, such as bacteria, leptospires, fungi, mycoplasma, rickettsiae, and viruses, have been proposed.

Kawasaki disease has clinical similarities to those of scarlet fever or toxic shock syndrome, both of which are caused by toxin-producing bacteria. These toxins are revealed to be superantigens capable of stimulating $\mathrm{T}$ cells carrying particular variable regions of the T-cell receptor $\beta$ chain. A study of peripheral blood T cells in KD patients showed that $T$ cells expressing $T$-cell receptor variable regions $V \beta 2$ and V $\beta 8$ were selectively expanded. ${ }^{14}$ These observations suggest that KD may be caused by a toxin (superantigen)-producing microorganism, including possibly a virus. Leung et al..$^{15}$ suggested that the expansion of $\mathrm{V} \beta 2+\mathrm{T}$ cells in most patients with KD may be caused by a new clone of toxic shock syndrome toxin-secreting Staphylococcus aureus or SPEB (streptococcal pyogenic exotoxin-B)- or SPEC (streptococcal pyogenic exotoxin-C)-producing group A streptococci from studies on bacterial culture from patients with $\mathrm{KD}^{15}$ However, other studies did not support this theory. ${ }^{16}$ Yoshioka et al. ${ }^{17}$ reported that SPEC induces activation and polyclonal expansion of V 32 - and V 36.5 -positive $\mathrm{T}$ cells, and that SPEC-induced activation of $\mathrm{T}$ cells may lead to the pathogenesis of KD. ${ }^{17}$

Yersinia pseudotuberculosis appears to be one of the etiologic agents of $\mathrm{KD}_{1}^{18}$ although only a small group of $\mathrm{KD}$ patients can be explained by this organism. Propionibacterium acnes was isolated from cervical lymph nodes of the patients, which produced the cytotoxic protein-like bacterial exotoxin, ${ }^{19,20}$ but its etiologic role remains to be determined. 
Epstein-Barr virus (EBV) occasionally induced clinical pictures mimicking those of $\mathrm{KD}^{21}$ and three of 37 patients with chronic active EBV infection developed coronary lesions. However, epidemiologic, serologic, and virologic studies do not support that EBV is a causative agent of KD. Rowley et al. ${ }^{22}$ reported that immunoglobulin A (IgA) plasma cells infiltrate the vascular wall as well as other tissues, such as the myocardium, respiratory tract, kidney, and pancreas in acute KD. They suggest a mucosal portal of entry of a conventional antigen; however, no specific microbe has been proposed as the etiologic agent. Recently, a possible association between novel human Corona virus (HcoV-NH) and KD was reported; however, further studies are necessary. ${ }^{23}$

Acute phase of KD is associated with markedly increased production of inflammatory cytokines. Tumor necrosis factor- $\alpha$ (TNF- $\alpha)$, interleukin- $1 \beta$ (IL-1 $\beta)$, and interferon- $\gamma($ IF- $\gamma$ ) induce activation antigens and adhesion molecules, such as endothelial leukocyte adhesion molecule-1 and intercellular adhesion molecule-1(ICAM-1) on endothelial cells, and TNF$\alpha$ and IF- $\gamma$ cause endothelial injury in in vitro studies. ${ }^{24}$ Leung and associates ${ }^{25}$ have proposed a hypothesis that antiendothelial antibodies cause endothelial injury in KD patients, based on the findings that circulating autoantibodies in KD were cytotoxic against human umbilical vein endothelial cells treated with TNF- $\alpha$, IL-1 $\beta$, or IF- $\gamma .{ }^{25}$ Coronary artery endothelial cells in KD patients expressed activation antigens in one study. ${ }^{26}$ Monocyte chemotactic and activating factor (MCAF or MCP-1) and TNF- $\alpha$ may have important roles in the pathogenesis of arteritis. ${ }^{27-29}$

Many hypotheses concerning the etiology and pathogenesis of KD have appeared; however, no host-microbe relationship specific to KD has been yet identified. Kawasaki disease patients are widely scattered in the community. They have self-limited disease, multiorgan involvement, and possible recurrence, and may have a tendency of epidemic development every 3 or 4 years. These findings suggest that this disease may be caused in some susceptible children by very common (ubiquitous) infectious agent(s).

\section{Clinical Presentations}

\section{Symptoms and Diagnosis of Kawasaki Disease}

The diagnosis of KD is made according to the diagnostic guidelines prepared by the Japanese Kawasaki Disease Research Committee listed in Table 41.1 because of the absence of a specific laboratory test. The principal diagnostic

TABLE 41.1. Diagnostic guideline of Kawasaki disease: $5^{\text {th }}$ revised edition, February 2002, Japanese Kawasaki Disease Research Committee, officially published by the Health and Warfare of Japanese Government

This is a disease of unknown etiology affecting most frequently infants and children under 5 years of age. The symptoms can be classified into two categories: principal symptoms and other significant symptoms or findings.

A. Principal Symptoms

1. Fever persisting 5 days or more (inclusive of those cases in whom the fever has subsided before the 5 th day in response to therapyl

2. Bilateral conjunctival congestion

3. Changes of lips and oral cavity: reddening of lips, strawberry tongue, diffuse injection of oral and pharyngeal mucosa

4. Polymorphous exanthema

5. Changes of peripheral extremities:

Initial stage: reddening of palms and soles, indurative edema

Convalescent stage: membranous desquamation from fingertips

6. Acute nonpurulent cervical lymphadenopathy

At least five items of 1 to 6 should be satisfied for diagnosis of Kawasaki disease. However, patients with four items of the principal symptoms can be diagnosed as having Kawasaki disease when a coronary aneurysm is recognized by two-dimensional echocardiography or coronary angiography.

B. Other Significant Symptoms or Findings

The following symptoms and findings should be clinically considered:

1. Cardiovascular: auscultation (heart murmur, gallop rhythm, distant heart sounds), ECG changes (prolonged PR/QT intervals, abnormal Q wave, low voltage, ST-T changes, arrhythmias), chest x-ray findings (cardiomegaly), 2D echo findings (pericardial effusion, coronary aneurysms), aneurysm of peripheral arteries other than coronary (axillary, etc.), angina pectoris or myocardial infarction

2. Gastrointestinal tract: diarrhea, vomiting, abdominal pain, hydrops of gallbladder, paralytic ileus, mild jaundice, slight increase of serum transaminase

3. Blood: leukocytosis with shift to the left, thrombocytosis, increased ESR, positive CRP, hypoalbuminemia, increased $\alpha_{2}$-globulin, slight decrease in erythrocyte and hemoglobin levels

4. Urine: proteinuria, increase of leukocytes in urine sediment

5. Skin: redness and crust at the site of BCG inoculation, small pustules, transverse furrows of the finger nails

6. Respiratory: cough, rhinorrhea, abnormal shadow on chest x-ray

7. Joint: pain, swelling

8. Neurological: pleocytosis of mononuclear cells in cerebrospinal fluid, convulsion, unconsciousness, facial palsy, paralysis of the extremities

Remarks:

1. For item 5 in principal symptoms, the convalescent stage is considered important.

2. Nonpurulent cervical lymphadenopathy is less frequently encountered (approximately 65\%) than other principal symptoms during the acute phase.

3. Male-to-female ratio is $1.3-1.5: 1$; patients under 5 years of age: $80-85 \%$; fatality rate: $0.1 \%$.

4. Recurrence rate: $2-3 \%$, proportion of siblings cases: $1-2 \%$.

5. Approximately $10 \%$ of the total cases do not fulfill five of the six principal symptoms, in which other diseases can be excluded and Kawasaki disease is suspected. In some of these patients coronary artery aneurysms (including so-called coronary artery ectasia) have been confirmed. 
criteria of $\mathrm{KD}$ are persistent fever, conjunctival injection, changes in the mucosa of the oropharynx, changes in the peripheral extremities, erythematous rash, and cervical lymphadenopathy. At least five of six principal symptoms should be present for a presumptive diagnosis of KD. However, some patients do not fulfill the classic criteria (Table 41.1), and their diagnosis has been called incomplete KD. Patients with incomplete KD have fewer than four of the six principal symptoms. Fever duration may be shorter than 5 days, particularly in those treated by gamma globulin within 5 febrile days. However, patients with four of these symptoms can be diagnosed when a coronary artery abnormality is recognized by two-dimensional (2D) echocardiographic findings or other imaging procedures, including dilatation or nonuniformity of arterial lumen and increased brightness of the arterial wall (Fig. 41.1). Incomplete KD is recognized in about $10 \%$ of all KD patients whereas they have higher incidence of coronary artery aneurysms (25\%). Infants less than 6 months of age and children over 7 years of age demonstrate atypical symptoms in some instances. Incomplete KD should be considered in children with unexplained fever for more than 4 days with three other principal symptoms. High C-reactive protein (CRP) (more than $3 \mathrm{mg} / \mathrm{dL}$ ), elevated erythrocyte sedimentation rate (ESR) (more than $40 \mathrm{~mm} / \mathrm{h}$ ), thrombocytosis (more than $400,000 / \mathrm{mm}^{3}$ ), white blood cell count (more than 15,000), and erythema and induration at the site of previous vaccination of bacille Calmette-Guérin (BCG) are helpful findings in diagnosing incomplete KD. In children over 7 years of age, high fever and neck lymphadenopathy are initial symptoms that occur several days after onset. Other significant symptoms and laboratory findings are listed in Table 41.1.

\section{Cardiovascular Spectrum}

The cardiovascular spectrum of 2180 patients with acute KD found in the past 31 years in the Kurume University Hospital in Kurume, Japan, is shown in Table 41.2. Among all patients, $14.9 \%$ were diagnosed as having coronary artery aneurysms. Beginning in 1992 we selected these patients for gamma globulin treatment; since then, the incidence of coronary artery aneurysms has declined to $8.7 \%$. Coronary artery lesions are the most important in KD patients; however, aneurysms in other arteries, such as the auxiliary, iliac, or renal arteries, were observed in $1.0 \%$ of the patients. Valvular heart disease appeared in about $1.5 \%$ of the patients. Myocardial infarction occurred in 24 patients, 10 of whom died. It is important to know that half of the fatal cases did not demonstrate any ischemic findings before the onset of myocardial infarction.

\section{Myocardial Infarction and Death}

The main cause of death in KD is acute myocardial infarction (MI). A study in 104 fatal cases of KD patients in Japan

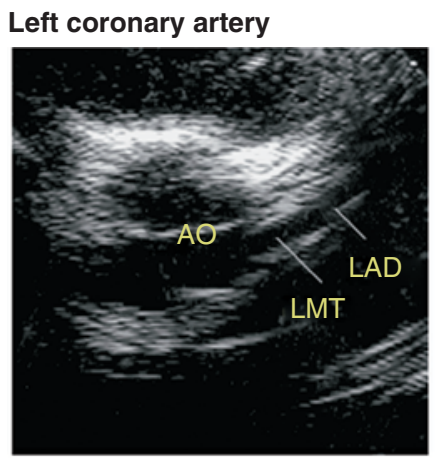

5 days of illness
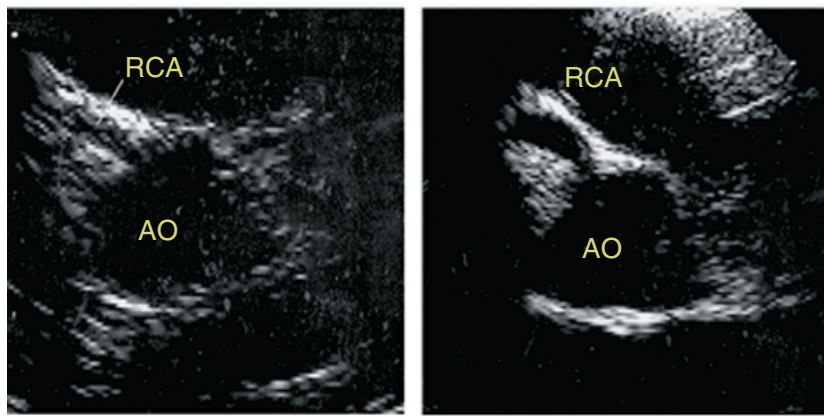

Right coronary artery

FIGURE 41.1. Serial two-dimensional echocardiography of the coronary artery in acute Kawasaki disease. Upper panel: Left main coronary artery. At 5 days of illness, the size of left main coronary artery looked to be normal. At 10 days of illness it demonstrated mild dilatation and the dense echo in the arterial wall, and gradually dilated afterward to develop to coronary aneurysm. Lower panel:
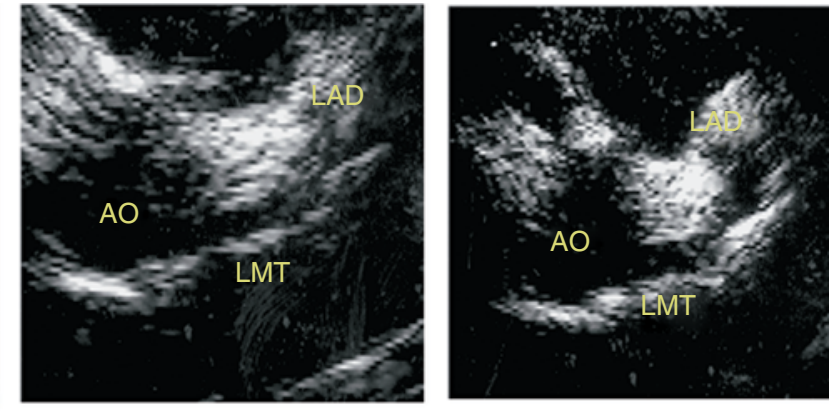

16 days

22 days
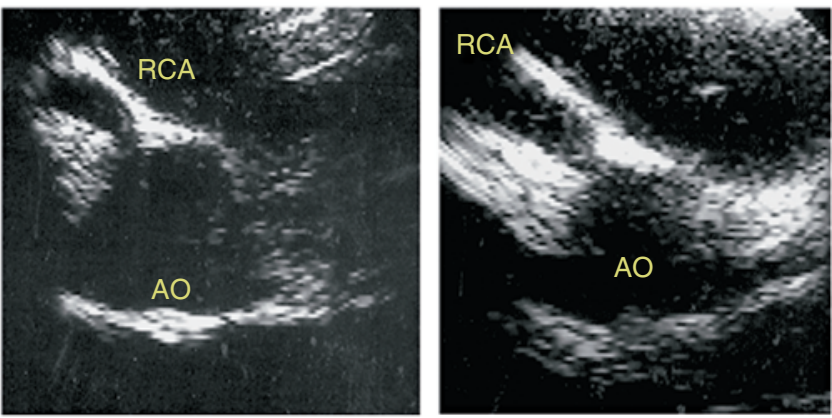

right main coronary artery. At 5 days of illness, the size of main right coronary artery looked normal; however, the arterial wall demonstrated the dense echo. At 10 days of illness, it demonstrated mild dilatation and had gradually developed to aneurysm. Ao, aorta; CX, circumflex branch; LAD, left anterior descending branch; LMT, left main trunk; RCA, right coronary artery. 
TABLE 41.2. Cardiovascular spectrum in Kawasaki disease (Kurume University, Kurume, Japan, 1973-2003)

\begin{tabular}{lc}
\hline Coronary artery & \\
$\quad$ Transient dilatation in acute stage & $282 / 1558(18.1 \%)$ \\
Coronary aneurysm & $325 / 2180(14.9)$ \\
$\quad$ 1973-1990, aspirin treatment & $253 / 1355(18.6)$ \\
$\quad$ 1991-2001, gamma globulin + aspirin & $72 / 825(8.7)$ \\
Systemic artery aneurysms & \\
$\quad$ Axillary, iliac, renal etc. & $22 / 2180(1.0)$ \\
Mitral regurgitation & $28 / 2180(1.3)$ \\
Aortic regurgitation & $5 / 2180(0.2)$ \\
Pericarditis or pericardial effusion & $241 / 1871(12.9)$ \\
Myocarditis & $617 / 2180(28.3)$ \\
Myocardial infarction & $24 / 2180(1.1)$ \\
Fatal cases & $10 / 2180(0.45)$ \\
\hline
\end{tabular}

suggests that $56.7 \%$ of the patients died of acute MI and $18 \%$ died of congestive heart failure caused by ischemia. Five patients died from rupture of coronary artery aneurysms. In the 1970 s the fatality rate was about $2 \%$ in Japan; in the $1990 \mathrm{~s}$ it declined to $0.2 \%$. We analyzed $195 \mathrm{KD}$ patients with MIs from a nationwide survey in Japan. ${ }^{30}$ Myocardial infarction mostly occurred within 1 year of illness; however, late deaths several years after acute KD have increased recently. It should be noted that death might occur at any time in the presence of obstructive lesions. Myocardial infarctions are relatively more frequent when the patient is sleeping or resting, and primary manifestations include shock symptoms, pallor, unrest, vomiting, and abdominal pain. Chest pain was more frequently recognized in survivors and in children over 4 years of age. Asymptomatic MIs were seen in $37 \%$ of patients. Twenty-two percent with MIs died; $16 \%$ of the survivors from a first attack had a second MI. Fatalities were $63 \%$ in a second attack and $83 \%$ in a third attack. From the coronary angiographic studies in patients with MIs, most of the patients who died had obstructions in the left main coronary artery or in both the right coronary artery and the left anterior descending artery. In survivors, one-vessel obstruction, particularly in the right coronary artery, was frequently recognized.

Early recognition and treatment of MIs are the critical problems. Recurrence of MI is observed in about $20 \%$ of the patients who have had a previous MI. Since the mortality of repeat $\mathrm{MI}$ is high, careful management is needed for such patients. Patients with complications after MI, such as ventricular aneurysm, papillary muscle dysfunction, heart failure, severe arrhythmias, and postinfarction angina, are managed by medical or surgical approaches.

\section{Systemic Artery Involvement}

Coronary artery lesions are the most important lesions in KD patients; however, aneurysms in other arteries were observed in $1.0 \%$ of the patients. ${ }^{31}$ Our angiographic study in 22 patients with systemic artery aneurysms demonstrated that systemic artery aneurysms were seen in axillary arteries in 18 cases, common iliac arteries in 16, internal iliac arteries in 12, renal arteries in six, mesenteric arteries in two, and internal thoracic arteries in two, respectively. One patient had large common iliac artery aneurysms associated with dilatation of aorta. Although prognosis of systemic artery aneurysms is generally favorable, renovascular hypertension may develop in a patient having a renal artery lesion, and intrathoracic arterial lesions may present problems at the time of coronary bypass surgery. There are some reports on digital gangrenous changes. ${ }^{32}$

\section{Valvular Heart Disease, Myocarditis, and Pericarditis}

Valvular heart disease occurs in $1 \%$ of the patients, mostly in mitral valves and rarely in aortic valves. We found acute mitral regurgitation in 28 patients among 2180 cases $(1.3 \%)$; this disappeared in half of the patients after a few months to several years. The etiology of this condition may be valvulitis or papillary muscle dysfunction caused by ischemia. ${ }^{33}$ Five patients presented with aortic regurgitation $(0.2 \%)$. It is noteworthy that aortic regurgitation appeared after acute or subacute stages of illness and had increased to severe regurgitation over several years in some patients. ${ }^{34}$ Pericarditis or pericardial effusions appeared in $12.9 \%$ of the patients in the acute phase that was mostly subclinical and disappeared within 1 or 2 weeks. Massive pericardial effusion and cardiac tamponade was rare. There have been no reports on its progression to chronic or constrictive pericarditis. Relatively mild myocarditis was observed in $28 \%$ of patients in the acute phase, especially in the first and second weeks of illness, irrespective of the presence of coronary artery aneurysms. Gallop rhythms, distant heart sounds, ST-T segment changes, and decreased voltage of $\mathrm{R}$ waves on electrocardiogram (ECG) may suggest the presence of myocarditis. In many instances, cardiac enzyme levels, such as creatine kinase $(\mathrm{CK})$, did not change significantly. Cardiomegaly and decreased ejection fractions of the left ventricle occurred, which were caused by myocarditis.

\section{Diagnostic Techniques}

The evaluation of the coronary artery lesions in $\mathrm{KD}$ in the acute stage of illness is important, even essential, and is usually done by 2D echocardiography (2D echo) and coronary angiography (CAG).

\section{Echocardiography}

Serial 2D echo studies are the most important and essential method to evaluate coronary artery aneurysms. The approach for $2 \mathrm{D}$ echo in KD is well described in the Japan KD Research Committee's standardization of diagnostic criteria and reporting of coronary artery lesions ${ }^{35}$ and the committee report of the American Heart Association (AHA). ${ }^{36}$ Some reports suggest that this approach may underestimate the true incidence of coronary artery dilatation. ${ }^{37,38}$ If a patient has abnormal findings on serial 2D echo studies, coronary angiography is indicated. ${ }^{39}$ 
The evaluation of the coronary artery morphology is particularly important in KD patients. The method for evaluation by echocardiography is well described in the AHA scientific statement on KD published in $2004 .^{40}$ The precordial short-axis segment is the standard approach for evaluation of the left main coronary artery, left anterior descending artery, and right coronary artery. The posterior descending artery can be evaluated by the apical four-chamber view. The middle segment of the right coronary artery is seen by the apical four-chamber view. In our experience using $2 \mathrm{D}$ echo, it is possible to correctly diagnose left main coronary artery aneurysms with $98 \%$ sensitivity and 95\% specificity compared to angiography. The echocardiographic evaluation for right coronary artery lesions was less sensitive. Falsenegative diagnoses were due mainly to the presence of isolated small peripheral coronary artery aneurysms, which were rare. From echocardiographic studies, it is evident that coronary artery dilatation appears at around 10 days of illness; approximately $40 \%$ of patients show coronary artery dilatation in this period. However, two thirds of these patients demonstrate a transient dilatation and regression within 3 to 5 weeks from the onset of illness (Fig. 41.2). This means that Kawasaki arteritis may cause the various degrees of coronary artery dilatation from mild transient dilatation, small or moderate-sized aneurysms, to giant aneurysms in more than half of the patients in the acute phase of the disease. ${ }^{41}$ Aneurysms are classified as saccular if the axial and lateral diameters are nearly equal or as fusiform if symmetric dilatation with gradual proximal and distal tapering is seen. If the coronary artery diameter looks larger than normal without segmental dilatation, the coronary artery is considered ectatic. The follow-up echocardiography is essential to evaluate such findings to assess whether these are ectatic changes or normal variations. We performed 2D echocardiography at the time of diagnosis, at 8 to 10 days after the onset of illness when coronary dilatation may appear, and at 2 and 8 weeks after the onset. The sizes of coronary artery aneurysms are classified as small $\mid<5 \mathrm{~mm}$ internal diameter), medium (5 to $8 \mathrm{~mm}$ ) and giant or large $(>8 \mathrm{~mm})$.

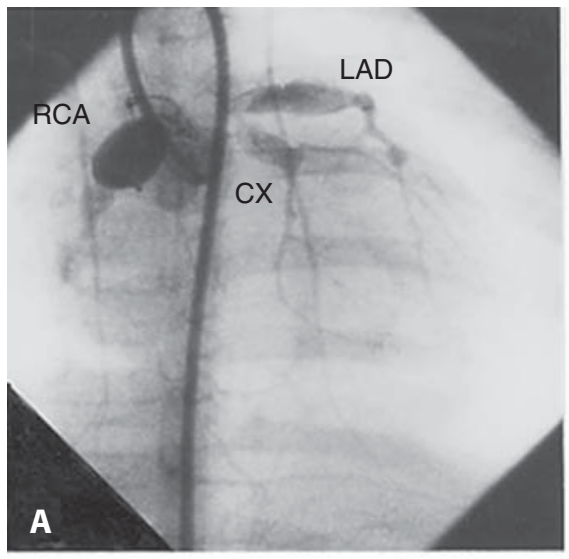

Acute KD (10 mo of age)
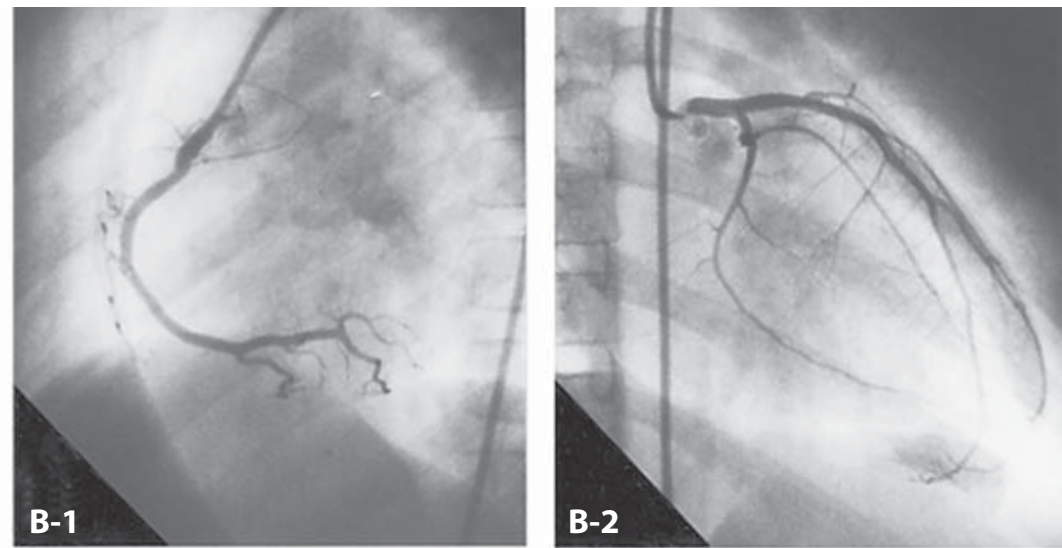

1 year of illness
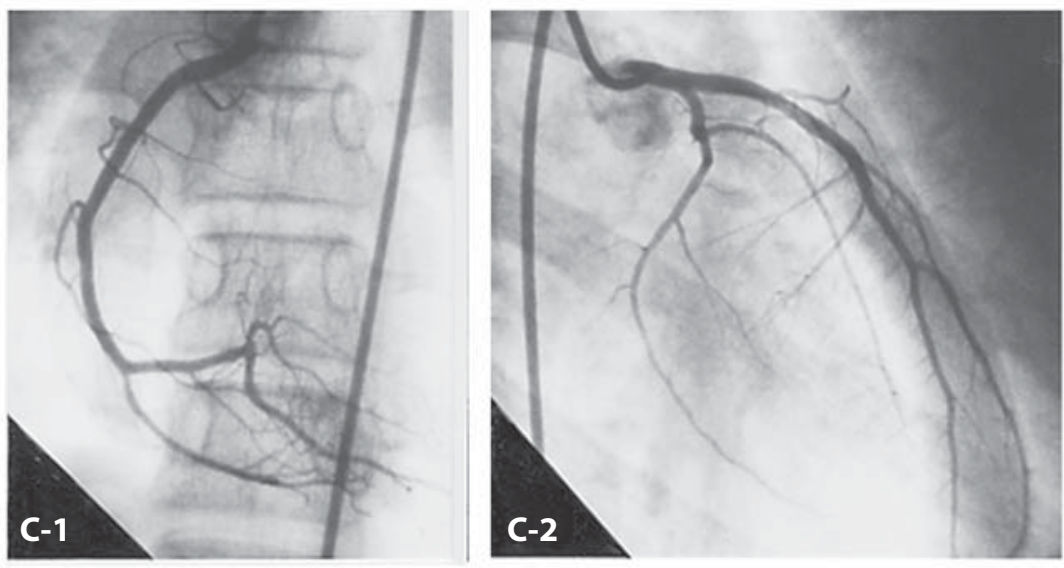

12 years later

FIGURE 41.2. Regression of coronary aneurysms. This patient was diagnosed with Kawasaki disease at age 11 months and developed aneurysms in both the right and left coronary arteries (A). One year and 2 months later, the follow-up coronary angiography demonstrated the disappearance of coronary aneurysms and no stenosis or irregular arterial lumen in the whole coronary artery (B-1, B-2). The follow-up coronary angiography 12 years later demonstrated no aneurysms, no stenosis, and no irregular arterial wall in both the left and right coronary arteries (C-1,C-2). 
Acute coronary thrombosis is diagnosed by serial 2D echo. At that time, thrombolytic treatment using urokinase or tissue-type plasminogen activator (t-PA) is indicated. ${ }^{42}$ The evaluation of stenotic lesions of the coronary artery by $2 \mathrm{D}$ echo is sometimes difficult; however, by using a high-frequency transducer and performing a careful examination, it may be possible. One looks for loss of uniformity of the lumen of arteries, irregular arterial walls, and dense echoes in the coronary arterial wall.

\section{Other Noninvasive Techniques}

Magnetic resonance imaging (MRI) may evaluate aneurysms in the proximal coronary artery and coronary flow profile. ${ }^{43}$ Ultrafast computed tomography $(\mathrm{CT})$ is also available to evaluate coronary artery aneurysms ${ }^{44}$; however, further studies on larger patient groups will be necessary.

\section{Coronary Angiography and Heart Catheterization}

Selective coronary angiography (CAG) is the most accurate method to define the presence and severity of coronary arterial abnormalities in KD patients. We have used specially designed catheters (three types) for selective CAG for infants, toddlers, and children that enable the CAG to be performed safely and successfully. ${ }^{39}$ The indications for CAG include the presence of abnormal 2D echo findings, the presence of symptoms or signs of ischemia, valvular regurgitation, evidence of cardiac dysfunction, and the use of intracoronary thrombolytic treatment. If the patient has severe coronary artery lesions, other systemic vascular involvement such as the auxiliary, iliac, renal, or intrathoracic arteries aneurysms should also be evaluated. Because the regression of coronary artery aneurysms and progression to stenotic lesions occur within 2 years from the onset of illness, follow-up CAG are essential, especially in patients having coronary aneurysms. ${ }^{31}$ The regression of coronary artery aneurysms is indicated when a follow-up CAG demonstrates normal findings in a patient who previously had coronary aneurysms. Aneurysms may disappear without any residual abnormality. This condition can be diagnosed by CAG; however, 2D echo may miss some mild abnormal findings, which subsequently progress to coronary artery disease. Coronary angiography is most important and essential for the evaluation of stenotic or obstructive lesions of coronary arteries and for evaluation of collateral circulation.

The clinical feasibility of intravascular ultrasound imaging for coronary arteries has been reported in patients who had KD to assess long-term pathology in vivo. ${ }^{45}$ At the site of regressed aneurysms, it has been shown that there is marked thickening of the intima. The portion of the coronary artery with normal angiographic findings shows normal findings except in the region near the regressed aneurysm where mild intimal thickening exists. Positive remodeling (or compensatory remodeling) may contribute to maintain the normal lumen with mild intimal thickening. ${ }^{46}$

\section{Evaluation for Myocardial Ischemia}

Because the morbidity and mortality of this disease primarily depend on the extent of associated coronary artery disease, it is particularly important to assess accurately the presence of myocardial ischemia during follow-up. The conventional methods have some limitations. Two-dimensional echocardiography can detect coronary aneurysm; however, its evaluation of stenotic lesions is not satisfactory. Similarly, electrocardiographic sensitivity for detection of myocardial ischemia is insufficient. Coronary angiography is an accurate method to assess coronary artery involvement; however, repeated evaluation is often difficult because it is an invasive technique. Although exercise stress tests can detect myocardial ischemia, and this noninvasive technique is easy to repeat, its sensitivity is not sufficient, even in patients with significant coronary stenosis, and it is difficult to perform in young children. In this regard, myocardial single photon emission computed tomography (SPECT) using pharmacologic stress (dipyridamole infusion) is considered to be the most accurate diagnostic method for identifying myocardial ischemia, especially in children in whom an exercise test cannot be performed. Because pharmacologic stress SPECT studies provide quantitative analysis, changes in the severity of myocardial ischemia can be detected when consecutive studies were performed. Kamiya ${ }^{47}$ reported that the sensitivity of detecting myocardial ischemia in patients having significant coronary artery stenoses was $>75 \%$. The most sensitive method was dipyridamole stress SPECT $185 \%$ sensitivity); the sensitivity of treadmill exercise tests was $<50 \%{ }^{48}$ Other stress tests reported in children with KD include nuclear perfusion scans with exercise, ${ }^{49}$ exercise echocardiography, ${ }^{50}$ stress echocardiography using dobutamine, ${ }^{51}$ and contrast echocardiography with dipyridamole stress. ${ }^{52}$

\section{Evidence-Based Treatment and Management}

Recommendations of treatment and management are described in an evidence-based grading scale of the American Medical Association. The levels of evidence are classified as follows: level A, randomized controlled trial (large scale); level B, randomized controlled trial (small sample); level C, prospective trial or large case series; level $\mathrm{D}$, retrospective analysis; level E, expert opinion or consensus; level M, metaanalysis; level $\mathrm{X}$, no evidence.

\section{Treatment of Acute Kawasaki Disease}

The combination of aspirin and high-dose gamma globulin is a major therapeutic strategy in acute $\mathrm{KD}$ at the present time $^{40}$ (level A evidence).

In 1984, high-dose gamma globulin treatment was reported to be effective by Furusho et al. ${ }^{53}$ in Japan (level A evidence). The treatment was confirmed by a U.S. multicenter randomized trial (level A). ${ }^{54}$ Both studies found that $400 \mathrm{mg} / \mathrm{kg}$ of gamma globulin may prevent coronary artery aneurysm formation. Gamma globulin is presently the most effective treatment; however, the optimal dose and indications for this treatment are still controversial. Some reports demonstrate a dose-depended effect with higher doses in single infusions being more effective (level $\mathrm{M}$ evidence). ${ }^{55} \mathrm{~A}$ large single dose of $2 \mathrm{~g} / \mathrm{kg}$ has been reported to be more effective (level A evidence). ${ }^{56,57}$ This treatment should be started 
within 10 days of illness, and if possible, within 7 days. It is uncertain whether very early treatment, that is, within 4 days of illness, is more effective (level C evidence). ${ }^{58}$

As mentioned before, coronary artery aneurysms develop in less than $20 \%$ of $\mathrm{KD}$ patients. If we can predict which patients may have coronary aneurysms and can select them for gamma globulin treatment, it might be more effective and reduce medical expenses. Harada's ${ }^{59}$ scoring system may be useful in selecting high-risk patients for gamma globulin treatment. Harada suggests the following criteria: (1) white blood cell count $>12,000 / \mathrm{mm}^{3}$; (2) platelet count $<350,000 /$ $\mathrm{mm}^{3}$; (3) CRP level $>3 \mathrm{mg} / \mathrm{dL}$; (4) hematocrit level <35\%; (5) serum albumin level $<3.5 \mathrm{mg} / \mathrm{dL}$; (6) age $<12$ months; and (7) male gender. The presence of at least four of the seven criteria is an indication for gamma globulin treatment.

The mechanisms of benefit from gamma globulin are uncertain; however, possible Fc-mediated mechanisms include downregulation of inflammatory cytokines; modulation of T/B-cell functions through binding to lymphocyte Fc receptors; and inhibition of activated complement to targets, such as vascular endothelium. As Fab-mediated mechanisms entail the neutralization of microbial toxins, antibodies against cytokines and anti-idiotypic reaction against autoantibodies are possible.

Another problem with gamma globulin treatment in KD is that coronary artery aneurysms may develop in some patients (about 13\% of gamma globulin treated patients) even using high-dose gamma globulin treatment. These patients may have a higher incidence of coronary aneurysms. Repeat gamma globulin treatment of $2 \mathrm{~g} / \mathrm{kg}$ or pulse therapy by highdose methylprednisolone $(30 \mathrm{mg} / \mathrm{kg}$ for 3 days) may be indicated (level D evidence). ${ }^{60}$

Aspirin is also important and provides antipyretic, antiinflammatory, and antiplatelet effects; however, optimal doses are still controversial (level C evidence). ${ }^{61,62}$ The AHA recommends high-dose aspirin (80 to $100 \mathrm{mg} / \mathrm{kg}$ ) and reducing the dose to 3 to $5 \mathrm{mg} / \mathrm{kg} /$ day after the child has been afebrile for 48 to 72 hours. High-dose aspirin and intravenous immunoglobulin (IVIG) appear to provide additive antiinflammatory effects. In Japan, lower doses of aspirin are prescribed, such as $30 \mathrm{mg} / \mathrm{kg}$ per day in two doses (middle dose) for 2 weeks and then reducing the dose to $5 \mathrm{mg} / \mathrm{kg}$ (level A evidence). ${ }^{61}$ Regarding the effects of aspirin on platelet aggregation in $\mathrm{KD}$, a lower dose $(30 \mathrm{mg} / \mathrm{kg})$ can successfully reduce the platelet aggregation that usually increases in the second or third week of illness. However, a higher dose of aspirin such as that used in collagen vascular disease, $100 \mathrm{mg} / \mathrm{kg}$ or more, did not have significant effects on platelet aggregation. ${ }^{63}$ Biosynthesis of thromboxane $B_{2}$ was completely blocked in both groups; however, plasma 6-keto prostaglandin $F_{2 \alpha}$, a metabolite of prostacyclin that has potent antiaggregatory effects on platelets, was also blocked in the high-dose group. These findings suggest that highdose aspirin may be disadvantageous for prevention of thrombosis in some of these patients. Liver dysfunction was seen more frequently in the high-dose group. In such cases, we discontinued aspirin and began flurbiprofen. It has been reported that the absorption of aspirin in the gastrointestinal tract is impaired and clearance may increase in the acute stages of $\mathrm{KD}$. The middle dose of aspirin may reduce aspirin intoxication, liver dysfunction, and gastrointestinal irritation, and have a lower risk for Reye's syndrome. For these reasons, we recommend $30 \mathrm{mg} / \mathrm{kg}$ in the acute stage and reduction to $5 \mathrm{mg} / \mathrm{kg}$ in convalescent stage (level $\mathrm{C}$ evidence).$^{40}$ However, the optimal dose of aspirin is still controversial.

Is steroid therapy beneficial? In the 1970 s, steroids (2 mg/ $\mathrm{kg}$ prednisone for 2 weeks) were frequently used for KD patients as a treatment for arteritis; however, there appeared to be a higher incidence of coronary artery aneurysms in patients receiving steroids, and also the regression of coronary aneurysms was less frequent. Furthermore, rupture of coronary aneurysm was frequently recognized in patients treated by steroids. ${ }^{64}$ Recently, studies reported that steroid pulse therapy for shorter periods with gamma globulin results in shorter fever periods (level B evidence). ${ }^{65}$ Steroid pulse therapy may effectively shorten the fever period and reduce acute inflammation; however, the longer use of steroids, such as the use of prednisone for 2 weeks, may have adverse effects on vascular remodeling, coronary aneurysm development, and Kawasaki arteritis. Frequent evaluation by echocardiography and long-term follow-up are necessary.

Other treatments that are available for acute KD patients are as follows: pentoxifylline (specific inhibitor of TNF- $\alpha$ messenger RNA transcription) ${ }^{66}$; Ulinastatin (inhibitor of neutrophil elastase $)^{67}$; plasma exchange for IVIG-refractory cases $^{68}$; infliximab (monoclonal antibody against TNF- $\left.\alpha\right)^{69}$; and cytotoxic agents such as cyclophosphamide or cyclosporine. ${ }^{70}$ As an antiplatelet agent, abciximab (platelet glycoprotein IIb/IIIa receptor inhibitor) may have efficacy for regression of coronary aneurysms. ${ }^{71}$ All these drugs may have some efficacy for treatment of KD patients; however, the control data are lacking, and small numbers of patients are reported (level E evidence).

\section{Long-Term Management}

Long-term management of patients with KD depends on the degree of coronary arterial involvement. Low-dose aspirin $(3$ to $5 \mathrm{mg} / \mathrm{kg} /$ day single dose) is the basic treatment in the convalescent phase, and it continues up to 6 to 8 weeks in patients without coronary artery abnormalities. If coronary arterial abnormalities are present, low-dose aspirin should be continued until they are resolved. Coronary angiography is recommended in children in whom cardiovascular abnormalities have been found by $2 \mathrm{D}$ echo. Since the risk of progression to ischemic heart disease is high, particularly in those with giant aneurysms, patients should be managed by pediatric cardiologists. We recommend the combination of aspirin and warfarin for patients with giant coronary aneurysms with controlled international normalized ratios (INRs) of 1.5 to 2.5 (level D evidence). Beta-blockade is indicated for patients with ischemic symptoms. Table 41.3 lists the recommendations for long-term management of patients with this disease.

When symptoms of acute MI are noted, the patient should be immediately hospitalized, given oxygen, and made to rest. Vital signs and electrocardiograms must be examined, and central venous pressure monitored. Since the main cause of 
TABLE 41.3. Therapeutic recommendation (evidence level described in the text)

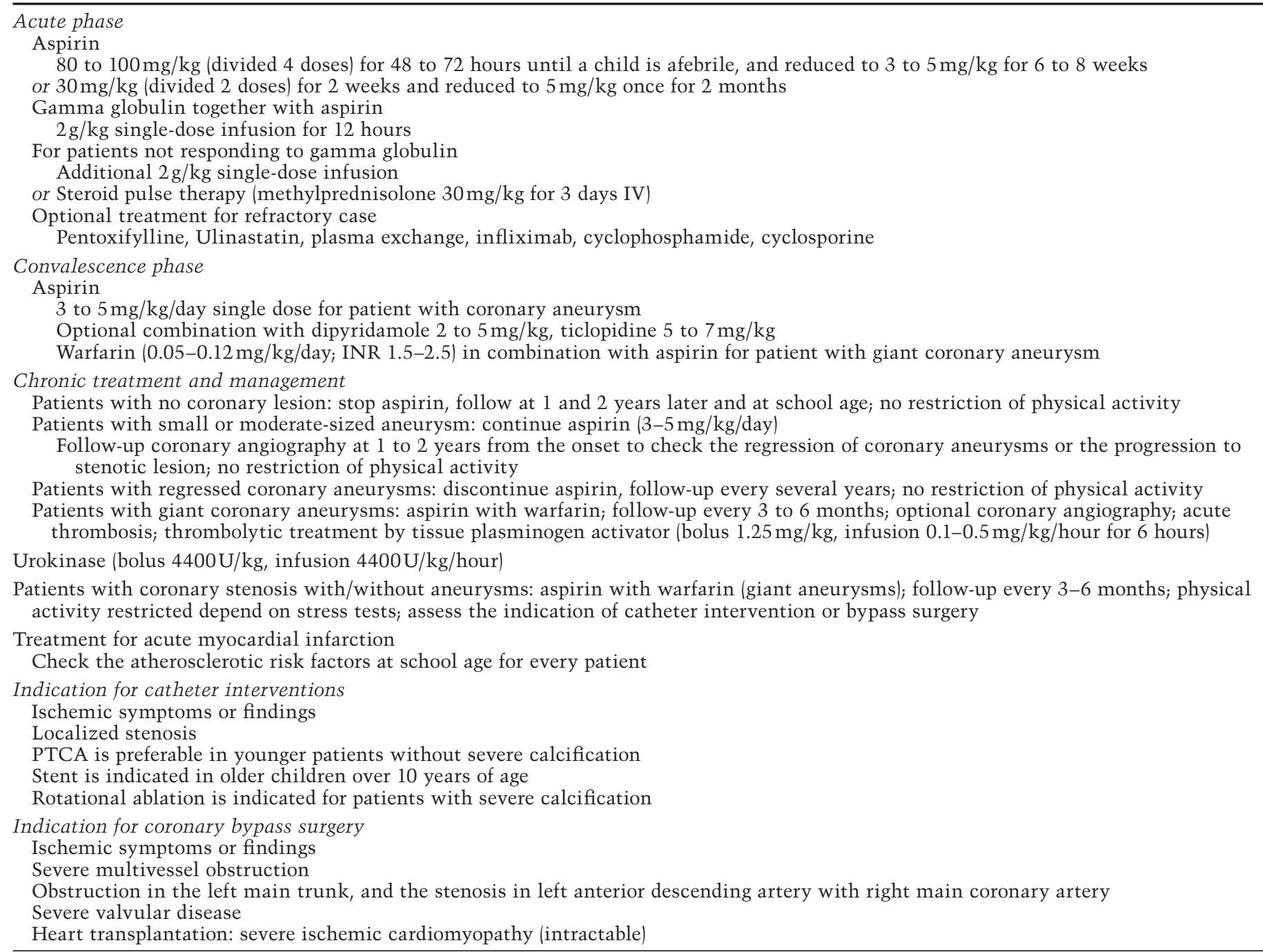

acute $\mathrm{MI}$ in $\mathrm{KD}$ patients is acute thrombotic occlusion in stenotic coronary aneurysms, thrombolytic treatment and anticoagulation therapy are important treatment for acute $\mathrm{MI}$, and they include intravenous t-PA, intracoronary infusion of urokinase, and heparin (Fig. 41.3). Intracoronary infusion of urokinase within 6 hours after the onset of MI is an effective treatment (level D evidence) ${ }^{42}$; however, t-PA can be used by intravenous infusion. Therefore, when acute MI occurs, t-PA should be used immediately intravenously and the patient referred to a cardiac center.

Interventional catheterization to treat coronary artery disease in KD patients has been performed in limited cases, so the long-term results are unknown. Balloon angioplasty is effective in some patients within 6 to 8 years from onset; however, with adult atherosclerotic coronary lesions, it was not so effective because of the elastic recoil and stiffness of the coronary artery. ${ }^{72}$ Recently, we have successfully performed coronary rotational ablation for long-term severe stenosis of the coronary artery in some patients that is frequently associated with severe calcification (level E evidence).$^{73}$ Stent implantation may be useful in some patients. The guidelines for catheter intervention in $\mathrm{KD}$ patients are published by the Japanese KD Research Committee (level C evidence). ${ }^{74}$ Catheter intervention may postpone or substitute for bypass surgery $^{75-77}$ (Fig. 41.4).

Coronary bypass surgery may be indicated for patients with extreme coronary artery lesions, and the result is generally favorable (level C evidence). ${ }^{78,79}$ Bypass grafting using an intrathoracic artery is recommended for left coronary artery bypass, because the long-term patency is more favorable $(3$ 

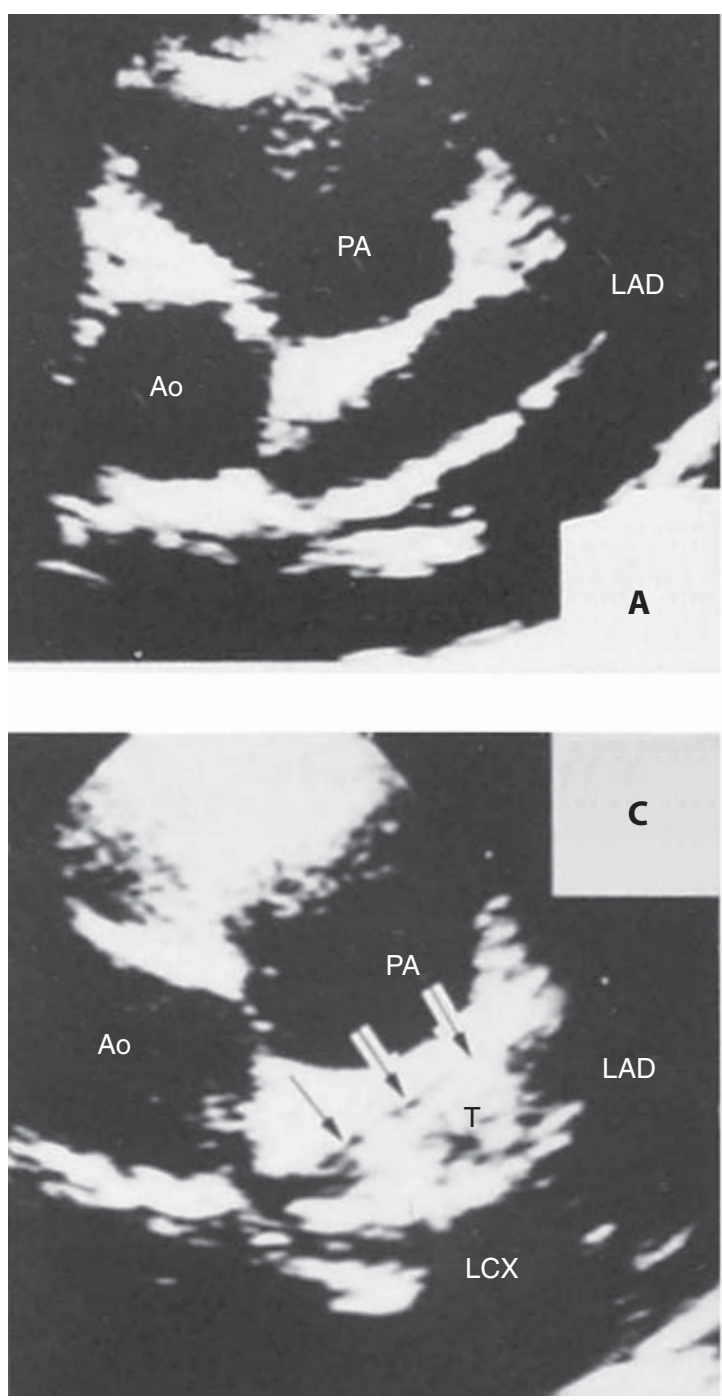

FIGURE 41.3. Myocardial infarction caused by acute thrombotic occlusion. This 3-year-old boy was diagnosed with Kawasaki disease and developed giant aneurysms in both the right and left coronary arteries as shown by two-dimensional echocardiography (A). Coronary angiography demonstrated a large aneurysm in the left main coronary artery and circumflex artery (LCX) and the stenosis in the anterior descending artery (B). No ischemic findings were recog-
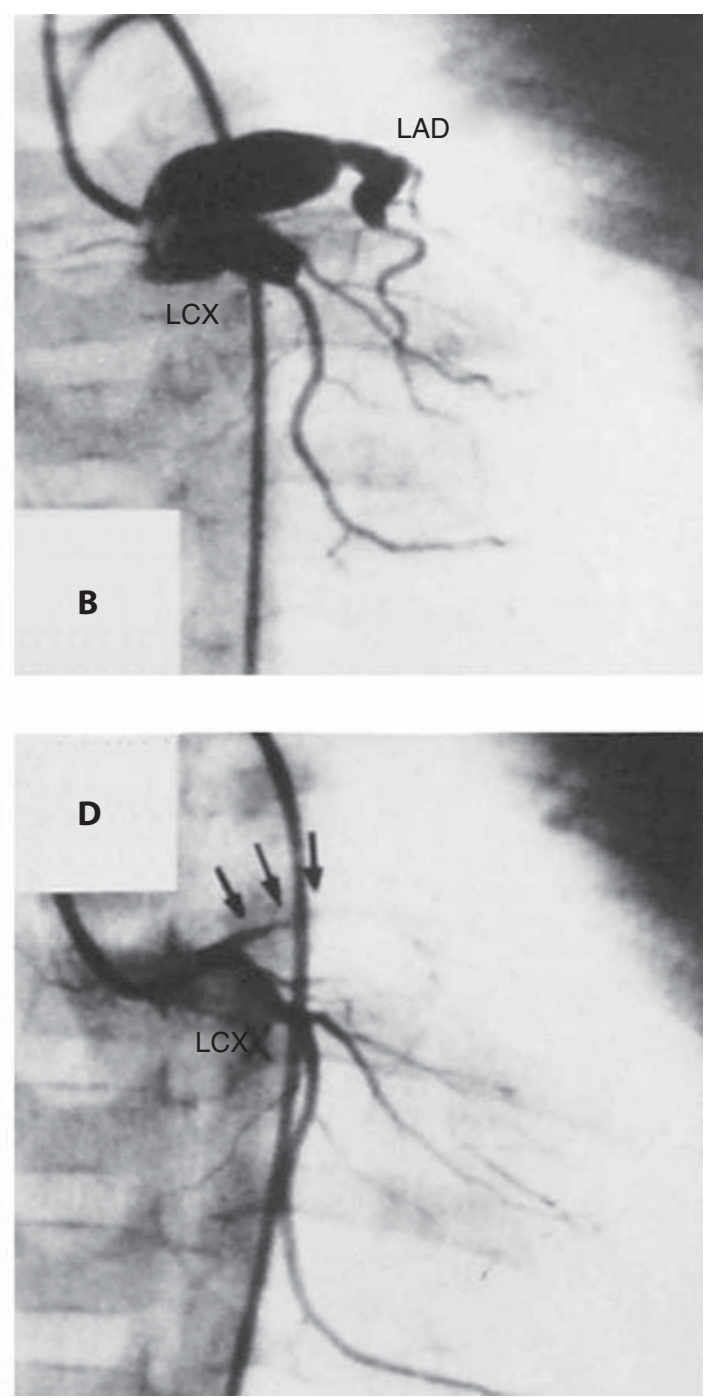

nized at that time. Three months later, he complained of frequent chest pain and vomiting. Echocardiogram during the acute myocardial infarction demonstrated complete occlusion in the left main coronary artery and the anterior descending artery $(\mathrm{C})$. The followup coronary angiography just after myocardial infarction demonstrated complete obstruction of the anterior descending artery (D). years, $77.1 \%$ ) compared to saphenous vein grafts (3 years, $52.8 \%$ ) (level D). The gastroepiploic artery is suitable for right coronary artery bypass grafting because it is usually large enough, even in young children. Surgical indications at the present time are as follows: (1) three-vessel obstruction; (2) severe stenosis of the left main coronary artery; and (3) severe stenoses in both left anterior descending artery and right coronary arteries. Even if patients are asymptomatic but demonstrate significant ischemic findings by exercise stress testing or myocardial scintigraphy, surgery may be indi- cated. ${ }^{80}$ A native, intrathoracic arterial graft is preferable, which can grow with age. ${ }^{81}$ Infants and children under 3 years of age present technical difficulties for bypass surgery. The viability of the myocardium should be evaluated by myocardial scintigraphy. Cardiac transplantation has been performed in several cases in the United States and the United Kingdom. ${ }^{82}$ It may be indicated in very rare instances in patients having severe diffuse myocardial fibrosis due to ischemia or previous multivessel bypass surgery with difficult revascularization. 

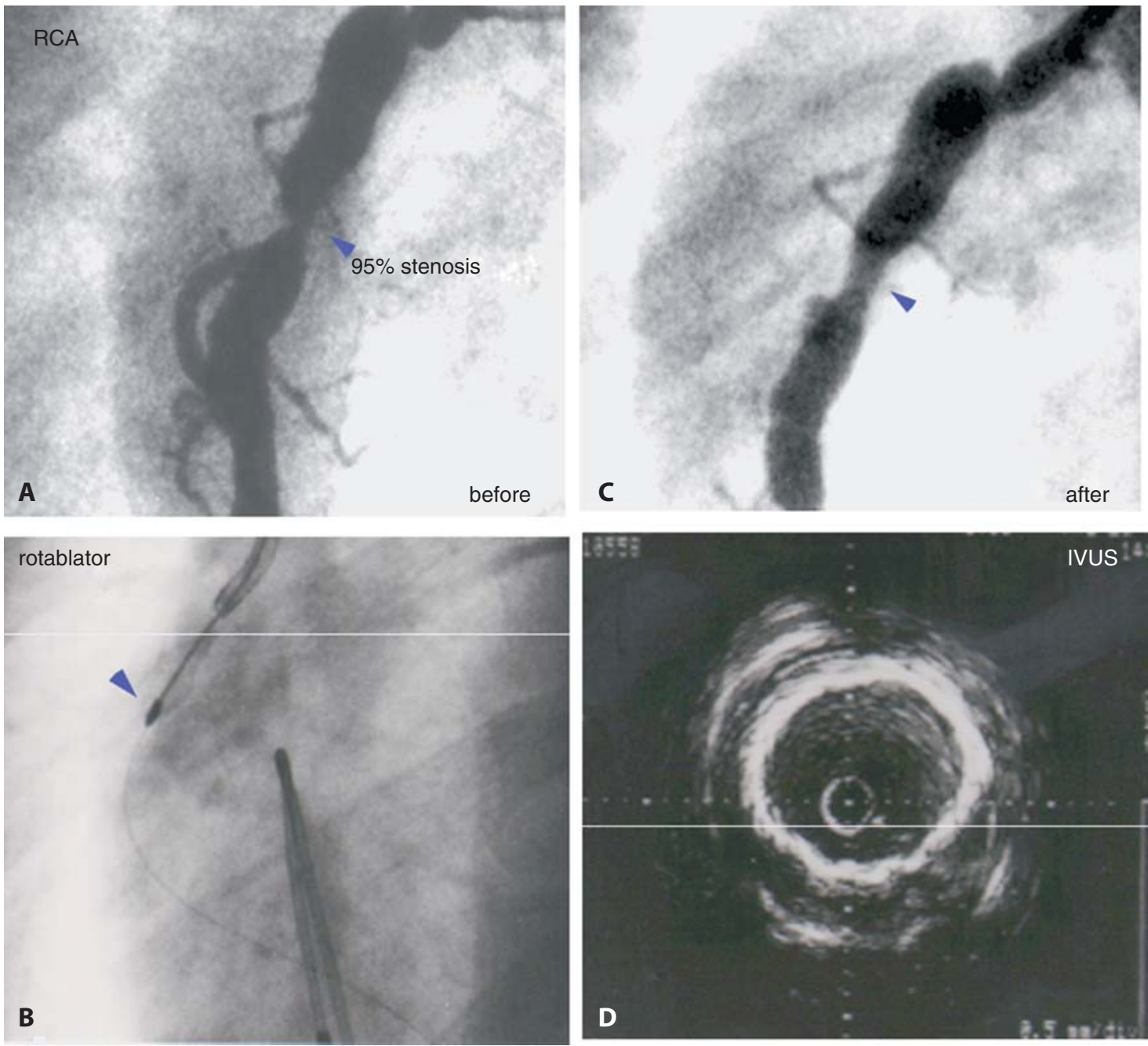

FIGURE 41.4. Catheter intervention for the stenotic lesion. Right coronary artery (RCA) angiography and intravascular ultrasound (IVUS) imaging in a 13-year-old boy with a stenotic RCA. (A) A 95\% localized stenosis of the RCA at the site of the proximal segment (arrow) before percutaneous rotational ablation (PTCRA) (B). (C)

Right coronary stenosis improved from $95 \%$ to $20 \%$ after PTCRA. (D) IVUS image at the site of the coronary artery stenosis after PTCRA showing calcification with high echogenicity and an acoustic shadow on the surface of the intima forming a ringlike structure.

\section{Prognosis and Long-Term Issues}

\section{Long-Term Consequences of Coronary Aneurysms}

The natural history or fate of coronary aneurysms is an important issue in KD patients ${ }^{83}$ (Fig. 41.5). We evaluated 594 consecutive patients with acute KD between 1973 and 1983, and this cohort was followed for 10 to 21 years. ${ }^{31}$ In all patients, we evaluated the coronary lesions by coronary angiography after the acute stage. One hundred forty-six patients $(24.6 \%)$ were diagnosed as having coronary artery aneurysms. A second angiography was performed 1 to 2 years later in all 146 patients who had previous coronary aneurysms, which demonstrated that $72(49.3 \%)$ had regression in the coronary artery aneurysms. It suggests that coronary artery aneurysms in KD patients show a strong tendency of regression. None of the patients with regression of coronary aneurysms had cardiac symptoms in the long-term follow-up periods,

and results of their ECGs, exercise stress tests, and left ventricular function were all within normal limits. In contrast, by 10 to 21 years after the onset of the illness, stenoses in the coronary aneurysms had developed in 28 patients. Myocardial infarctions occurred in 11 patients, five of whom died. From this study, it is estimated that about $4 \%$ of patients with KD may develop ischemic heart disease.

When does this condition occur? We studied the time and the incidence of regression or progression to obstructive lesions from the onset of KD using the Kaplan-Meier life table method. Regression of coronary aneurysms mostly occurred within 2 years from the onset of illness, whereas the obstructive lesions developed in 2 years and gradually increased over several years.

We investigated various factors that could affect the prognosis of coronary aneurysms. ${ }^{84}$ The risk factors for coronary aneurysms to develop into ischemic heart disease are as follows: (1) aneurysm diameter more than $8 \mathrm{~mm}$; (2) large 


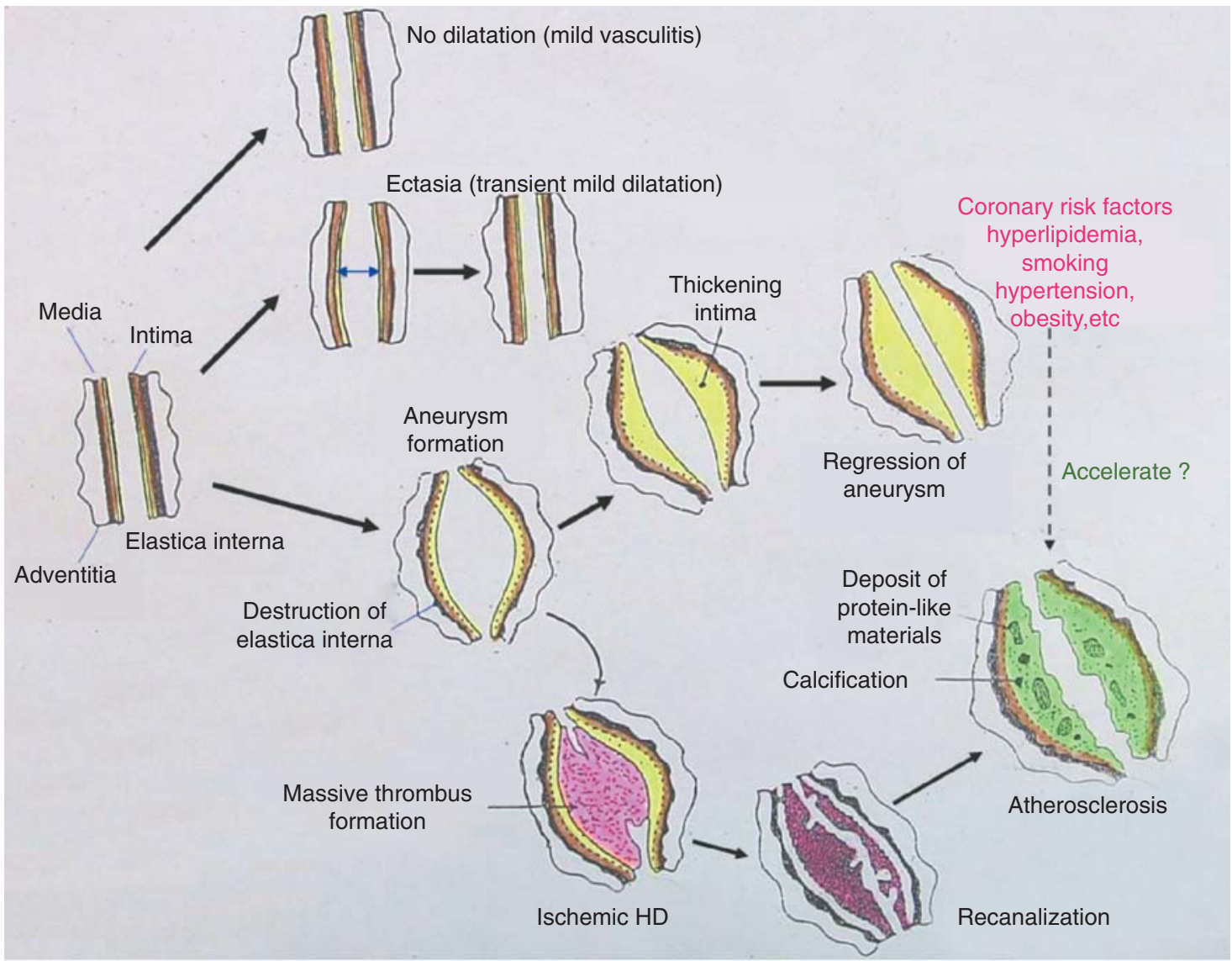

FIGURE 41.5. Pathologic sequence of coronary aneurysms. HD, heart disease.

diffuse or saccular-type aneurysms; (3) prolonged fever for $>21$ days; and (4) age at onset $>2$ years. In the 26 patients with giant coronary aneurysms, stenotic lesions developed in 12, and no regression occurred in our follow-up study. Thus, giant coronary aneurysms often progress to ischemic heart disease. The incidence of giant coronary aneurysms was $17.8 \%$ in the patients with coronary aneurysms and $4.4 \%$ among all KD patients in our series.

The biologic mechanism of regression of aneurysms is marked proliferation of intima without massive thrombus formation, and the intima consists of rich, smooth muscle cells and well-regenerated endothelium. ${ }^{85}$ Hemodynamic forces may regulate such arteries to maintain adequate lumen. It is uncertain whether intimal thickening eventually develops into obstructive lesions. However, in our 10- to 21-year follow-up study, none of the patients who had regressed aneurysms developed ischemic heart disease. Our experience indicates that stenotic lesions do not develop, at least within one or two decades, in patients with complete regression of coronary artery aneurysms.

\section{Impact of Kawasaki Disease on Overall and Cardiovascular Mortality}

Chronologic changes in mortality and fatality rates from KD patients were studied by Hayakawa et al. ${ }^{86}$ by analyzing 679 death cases in Japan between 1972 and 1998. The maleto-female ratio of the number of deaths was 2.07. The age-specific mortality rate was highest in infants under 1 year of age. The case fatality rate has decreased annually, declining from $2.23 \%$ in 1973 to as low as $0.02 \%$ in 2002 , which may be due mostly to the improvement of cardiac evaluation and treatment, particularly by gamma globulin and aspirin.

Nakamura et al. ${ }^{87}$ followed a cohort of 6576 patients for 12.6 years to determine whether patients with KD have a higher death rate than an age-matched healthy population. In this cohort, 27 (19 males, 8 females) died, and 12 died from myocardial infarction or mitral regurgitation from KD. Eight of 12 died within 2 months of illness. The other causes of death were as follows: four died of congenital heart failure, four died of neoplasms, three died of other diseases, and five died of injuries or accidents. In contrast to the high mortality rate during the acute $\mathrm{KD}$, the mortality rate was not elevated for those without cardiac sequelae after the acute phase. On the other hand, high but not significant mortality was observed for male patients with cardiac sequelae after the acute phase of the disease. This study has only a 12 -year follow-up, so a longer follow-up observation in the adult may be necessary (Fig. 41.6). 


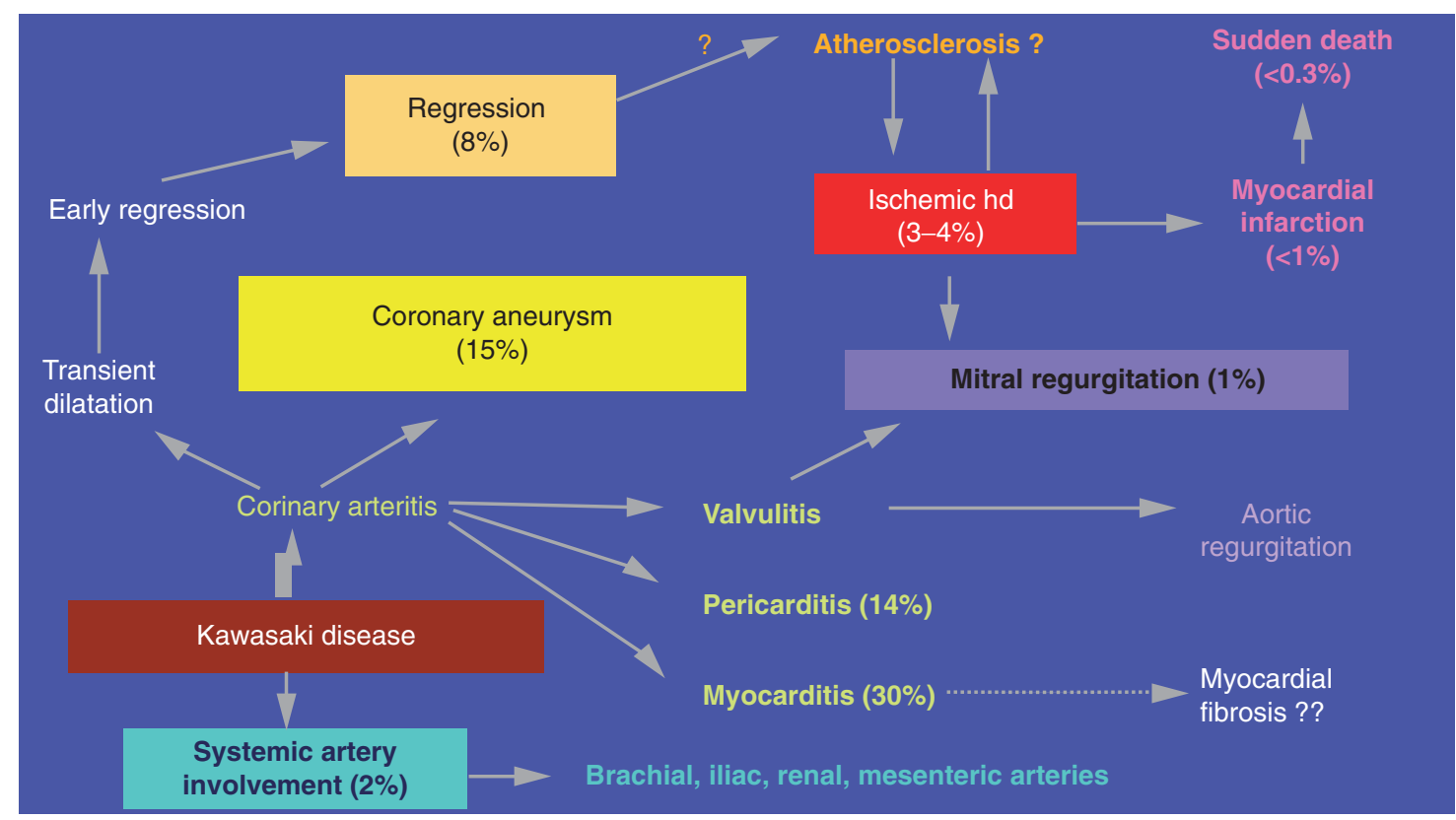

FIGURE 41.6. The cardiovascular spectrum and its natural history.

\section{Kawasaki Arteritis May Be an Atherosclerotic Risk Factor}

The pathology of coronary arteritis in KD patients several years after onset demonstrates marked intimal proliferation and, in some patients, calcific deposits of protein-like material and hyalinized degeneration in the thickened intima. Intravascular ultrasound studies of the coronary arteries after KD demonstrated marked thickening of intima and calcification in the coronary aneurysms. ${ }^{45}$ These findings are similar to arteriosclerotic lesions. Suzuki et al. ${ }^{88,89}$ reported that the active expression of various growth factors was observed in the coronary arteries in the late phases of $\mathrm{KD}$, suggesting that active vascular remodeling continues several years after the onset of the disease. However, the important issue is whether these coronary artery lesions develop into atherosclerotic coronary artery disease in adulthood.

We studied the distensibility of the coronary arterial wall by using intracoronary infusion of isosorbide dinitrate ${ }^{90}$ and endothelial function by intracoronary infusion of acetylcholine. ${ }^{91,92}$ Mitani and Dhillon's group ${ }^{93,94}$ reported that endothelial dysfunction was observed even in the normal artery in KD patients. Pathology of the coronary arteries in adults after KD demonstrated premature atherosclerotic changes. ${ }^{95}$ Mitani et al. ${ }^{96}$ suggested that high-sensitivity CRP was elevated in the late coronary sequelae in KD. These results suggest that the coronary arteries in the late KD patients may have a potential coronary risk factor. ${ }^{96}$

\section{Adult Coronary Artery Disease Due to Childhood Kawasaki Disease}

It has now been more than 35 years since the first description of KD as a new clinical entity, and the early KD children are now adults. We surveyed these adults to investigate any coronary sequelae that might be due to their earlier $\mathrm{KD}$ and reported 21 such cases and their cardiac conditions. ${ }^{97}$ Most of these 21 patients were diagnosed as having fever of unknown origin, sepsis, or pneumonia because they suffered from suspected Kawasaki disease before Dr. Kawasaki's first description in 1968. Most of the patients had ischemic symptoms or signs, including acute or old myocardial infarction or angina pectoris, two or three decades after the onset of suspected KD. Some of the patients demonstrated mitral regurgitation or arrhythmias. Almost all patients had multiple coronary artery aneurysms with obstruction or stenosis and with severe calcification of the arteries. Coronary aneurysms of unknown etiology were also reported in 109 patients who had no history of KD. These patients were mostly free from the ischemic symptoms, but had abnormal Q waves on their ECGs or calcification of the coronary arteries on chest $\mathrm{x}$-ray. We suspect that these findings in large or small part may be caused by childhood KD.

Our study suggests that some patients with coronary artery sequelae of KD have progressed into young adulthood with ischemic heart disease. The suspected findings for recognizing this condition are important. A past history in infancy or childhood is important. Abnormal Q waves or mitral regurgitation of unknown origin in the younger adults is also important. Coronary angiographic findings are essential for recognition as these are multiple aneurysms frequently associated with calcification. ${ }^{98}$ Familial hypercholesterolemia or collagen vascular disease should be excluded. After our report, many case reports on adult coronary artery disease attributed to childhood KD have appeared that were reviewed by Burns.99 The coronary artery sequelae of $\mathrm{KD}$ may be an important cause of ischemic heart disease in young adults, particularly those under 40 years of age. Cardiologists should recognize this condition and include the sequelae of KD in the differential diagnosis of early-onset coronary artery disease in adults. 


\section{Guidelines}

The following is a list of available KD guidelines in the literature:

1. Research Committee on Kawasaki Disease. ${ }^{35}$

2. Guideline for treatment and management of cardiovascular sequelae in Kawasaki disease. ${ }^{80}$

3. American Heart Association guidelines for diagnosis and treatment of Kawasaki disease. ${ }^{100}$

4. Guidelines for long-term management of patients with Kawasaki disease. ${ }^{36}$

5. Guidelines for catheter intervention in coronary artery lesion in Kawasaki disease. ${ }^{74}$

6. Guidelines for diagnosis and management of cardiovascular sequelae in Kawasaki disease. ${ }^{101}$

7. American Heart Association's diagnosis, treatment, and long-term management of Kawasaki disease. ${ }^{40}$

\section{Summary}

Kawasaki disease is a relatively common acute febrile illness with mucocutaneous involvement that affects infants and young children in Japan and the Orient. It is an arteritis syndrome mostly affecting small and medium-sized arteries, particularly coronary arteries. Coronary artery aneurysms occur in $10 \%$ to $20 \%$ of patients and may cause myocardial infarction, sudden death, and coronary artery disease. In Japan, North America, and Western Europe, Kawasaki disease has replaced rheumatic fever as the leading cause of acquired heart disease in children. Furthermore, it may develop into coronary artery disease in adults. Long-term follow-up studies are important to clarify the long-term prognosis in pediatric through adult patients.

\section{References}

1. Kawasaki T, Kosaki F, Okawa S, Shigematsu I, Yanagawa H. A new infantile acute febrile mucocutaneous lymph node syndrome prevailing in Japan. Pediatrics 1974;54:271-276.

2. Kato H, Akagi T, Sugimura T, et al. Kawasaki disease. Coronary Artery Disease 1995;6:194-206.

3. Kawasaki T. Acute febrile mucocutaneous syndrome with lymphoid involvement with specific desquamation of the fingers and toes in children. Jpn J Allerg 1967;16:178-222.

4. YamamotoT, Kimura J. A case report of acute febrile MCLS complicated with myocarditis (in Japanese). Shonika Rinsho $1968 ; 21: 336-339$

5. Kato H, Koike S, Yamamoto M, Ito Y, Yano E. Coronary aneurysms in infants and young children with acute febrile mucocutaneous lymph node syndrome. J Pediatr 1975;86:892-898.

6. Shibuya N, Shibuya K, Kato H, Yanagisawa M. Kawasaki disease before Kawasaki at Tokyo University Hospital. Pediatrics 2002;110:e17.

7. Landing $\mathrm{BH}$, Larson EJ. Are infantile periarteritis nodosa with coronary artery involvement and fetal mucocutaneous lymph node syndrome the same? Comparison of 20 patients from North America with patients from Hawaii and Japan. Pediatrics 1977;59:651-662.

8. Yanagawa $H$, Yashiro $M$, Nakamura $T$, Kawasaki $T$, Kato $H$. Results of twelve nationwide epidemiological incidence surveys of Kawasaki disease in Japan. Arch Pediatr Adolesc Med 1995; 149:779-783.
9. Nakamura Y, Yanagawa H. The worldwide epidemiology of Kawasaki disease. Prog Pediatr Cardiol 2004;19:99-108.

10. Chang RK. Hospitalization for Kawasaki disease among children in the United States, 1988-1997. Pediatrics 2002;109:e87.

11. Fujiwara H, Hamashima Y. Pathology of the heart in Kawasaki disease. Pediatrics 1978;61:100-107.

12. Naoe S, Shibuya K, Takahashi K. Wakayama M, Masuda H, Tanaka M. Pathological observations concerning the cardiovascular lesions in Kawasaki disease. Cardiol Young 1991;3:212220.

13. Yutani C, Okano K, Kamiya T, et al. Histopathological study on right endomyocardial biopsy of Kawasaki disease. Br Heart J 1980;43:589-592.

14. Abe J, Kotzin BL, Jujo K, et al. Selective expansion of $\mathrm{T}$ cells expressing T-cell receptor variable regions Vbeta2 and Vbeta8 in Kawasaki disease. Proc Natl Acad Sci USA 1982;89:40664070 .

15. Leung DYM, Meissner HC, Fulton DR, Murray D, Kotzin BL, Schllevert PM. Toxic shock syndrome toxin-secreting staphylococcus aureus in Kawasaki syndrome. Lancet 1993;342:13851388

16. Sakaguchi M, Kato H, Nishiyori A, Sagawa K, Itoh K. Characterization of CD4+ T helper cells in patients with Kawasaki disease: Preferential production of TNF-alpha by Vbeta 2 and Vbeta8 CD4+ T helper cells. Clin Exp Immunol 1995;99:276282.

17. Yoshioka T, Matsutani T, Toyosaki-Maeda T, et al. Relation of streptococcal pyrogenic exotoxin $\mathrm{C}$ as a causative superantigen for Kawasaki disease. Pediatr Res 2003;53:403-410.

18. Sato K, Ouichi K, Taki M. Yersinia pseudotuberculosis infection in children, resembling Izumi fever and Kawasaki syndrome. Pediatr Infect Dis J 1983;2:123-126.

19. Kato H, Fujimato $T$, Inoue $\mathrm{O}$, et al. Variant strain of Propionibacterium acnes: a clue to the aetiology of Kawasaki disease. Lancet $1983 ; 2: 1383-1388$.

20. Tomita S, Kato H, Fujimoto T, et al. Cytopathogenic protein in filtrates from cultures of Propionibacterium acnes isolated from patients with Kawasaki disease. Br. Med J 1987;295:1229_ 1232.

21. Kikuta H, Mizuno F, Osato T. Kawasaki disease and an unusual primary infection with Epstein-Barr virus. Pediatrics 1984; 73:413-414.

22. Rowley AH, Shulman ST, Spike BT, Mask CA, Baker SC. Oligoclonal IgA response in the vascular wall in acute Kawasaki disease. J Immunol 2001;166:1334-1343.

23. Esper F, Shapiro ED, Welbel C, Ferguson D, Landry ML, Kahn JS. Association between a novel human Coronavirus and Kawasaki disease. J Infect Dis 2005;191:499-502.

24. Furukawa S, Matsubara $T$, Jujoh $\mathrm{K}$, et al. Peripheral blood monocyte/macrophages and serum tumor necrosis factor in Kawasaki disease. Clin Immunol Immunopathol 1988;48:247251.

25. Leung DYM, Collins T, LaPierre LA, Geha RS, Pober JS. Immunoglobulin $M$ antibodies present in the acute phase of Kawasaki syndrome lyse cultured vascular endothelial cells stimulated by gamma interferon. J Clin Invest 1986;77: $1428-1435$

26. Terai M, Kohno Y, Nanba M, et al. Class II antigen expression in the coronary artery endothelium in Kawasaki disease. Hum Pathol 1990;21:231-234.

27. Yasukawa K, Terai M, Shulman ST, et al. Systemic production of vascular endothelial growth factor in fms-like tyrosine kinase-1 receptor in acute Kawasaki disease. Circulation 2002; 105:766-769.

28. Furukawa S, Matsubara T, Umezawa Y, Okamura K, Yabuta K. Serum levels of p60 soluble tumor necrosis factor receptor during acute Kawasaki disease. J Pediatr 1994;124:721-725. 
29. Lin CY, Lin CC, Hwang B, Chiang B. Serial changes of serum interleukin-6, interleukin-8, and tumor necrosis factor alpha among patients with Kawasaki disease. J Pediatr 1992;121: 924-926.

30. Kato H, Ichinose E, Kawasaki T. Myocardial infarction in Kawasaki disease: clinical analyses in 195 cases. J Pediatr 1986;108:923-927.

31. Kato H, Sugimura T, Akagi T, et al. Long-term consequences of Kawasaki disease: A 10- to 21-year follow-up study of 594 patients. Circulation 1996;94:1379-1385.

32. Tomita S, Chung K, Mas M, Gidding S, Shulman ST. Peripheral gangrene associated with Kawasaki disease. Clin Infect Dis 1992;14:121-126.

33. Akagi $T$, Kato $H$, Inoue $O$, Sato $N$. Valvular heart disease in Kawasaki syndrome: Incidence and natural history. Am Heart J 1990;120:366-372.

34. Gidding SS, Shulman ST, Ilbawi M, Crussi F, Duffy CE. Mucocutaneous lymph node syndrome (Kawasaki disease): delayed aortic and mitral insufficiency secondary to valvulitis. J Am Coll Cardiol 1986;7:894-897.

35. Research Committee on Kawasaki disease. Report of Subcommittee on Standardization of Diagnostic Criteria and Reporting of Coronary Artery Lesions in Kawasaki Disease. Tokyo, Japan: Ministry of Health and Welfare, 1984.

36. Dajani AS, Taubert KA, Takahashi $M$, et al. Guidelines for long-term management of patients with Kawasaki disease, Committee Report of Council on Cardiovascular Disease in the Young, American Heart Association. Circulation 1994;89: 916-922.

37. de Zorzi A, Colan SD, Gauvreau K, et al. Coronary artery dimensions may be misclassified as normal in Kawasaki disease. J Pediatr 1998;133:254-258.

38. Kurotobi S, Nagai T, Kawakami N, et al. Coronary diameter in normal infants, children and patients with Kawasaki disease. Pediatr Int 2002;44:1-4.

39. Kato H, Ichinose E, Yoshioka $F$, et al. Fate of coronary aneurysms in Kawasaki disease: serial coronary angiography and long-term follow-up study. Am J Cardiol 1982;49:17581766.

40. Newburger JW, Takahashi M, Gerber MA, et al. Diagnosis, treatment, and long-term management of Kawasaki disease. American Heart Association Scientific Statement. Circulation 2004;110:2747-2771.

41. Kato H. Cardiovascular complications in Kawasaki disease: coronary artery lumen and long-term consequences. Prog Pediatr Cardiol 2004;19:137-145.

42. Kato $\mathrm{H}$, Inoue $\mathrm{O}$, Ichinose $\mathrm{E}$, Akagi $\mathrm{T}$, Sato N. Intracoronary urokinase in Kawasaki disease: treatment and prevention of myocardial infarction. Acta Paediatr Jpn 1991;33:27-35.

43. Greil GF, Stuber M, Botnar RM, et al. Coronary magnetic resonance angiography in adolescent and young adults due to Kawasaki disease. Circulation 2002;105:908-911.

44. Frey EE, Matherne GP, Mahony LT, Sato Y, Stanford W, Smith WL. Coronary artery aneurysms due to Kawasaki disease: diagnosis with ultrafast CT. Radiology 1988;167:725-726.

45. Sugimura $\mathrm{T}$, Kato $\mathrm{H}$, Inoue $\mathrm{O}$, et al. Intravascular ultrasound of coronary arteries in children: assessment of the wall morphology and the lumen after Kawasaki disease. Circulation 1994;89:258-265.

46. Glagov S, Weinsenberg E, Kolettis G, et al. Compensatory enlargement of human coronary arteries. N Engl J Med 1987; 316:1371-1375

47. Kamiya T. How to evaluate the myocardial ischemic in Kawasaki disease. In: Kato $\mathrm{H}$, ed. Kawasaki Disease. Amsterdam: Elsevier, 1995:447-450.

48. Fukuda $T$, Akagi $T$, Ishibashi $M$, Inoue $O$, Sugimura $T$, Kato $H$. Noninvasive evaluation of myocardial ischemia in Kawasaki disease: comparison between dipyridamole stress thallium imaging and exercise stress testing. Am Heart J 1998;135: 482-487.

49. Kondo C, Hiroe M, Nakanishi T, Takao A. Detection of coronary artery stenosis in children with Kawasaki disease. Circulation 1989;80:615-624.

50. Pahl E, Sehgal R, Chrystof D, et al. Feasibility of exercise stress echocardiography for the follow-up of children with coronary involvement secondary to Kawasaki disease. Circulation 1995; 91:122-128.

51. Noto N, Ayusawa M, Karasawa K, et al. Dobutamine stress echocardiography for detection of coronary artery stenosis in children with Kawasaki disease. J Am Coll Cardiol 1996;27: 1251-1256.

52. Ishii M, Himeno W, Sawa M, et al. Assessment of the ability of myocardial contrast echocardiography with harmonic power Doppler imaging to identify perfusion abnormalities in patients with Kawasaki disease at rest and during dipyridamole stress. Pediatr Cardiol 2002;23:192-199.

53. Furusho K, Kamiya T, Nakano H, et al. High-dose intravenous gamma globulin for Kawasaki disease. Lancet 1984;2:10551058.

54. Newburger JW, Takahashi M, Burns JC, et al. The treatment of Kawasaki syndrome with intravenous gamma globulin. N Engl J Med 1986;315:341-347.

55. Terai M, Shulman S. Prevalence of coronary artery abnormalities in Kawasaki disease is highly dependent on gamma globulin dose but independent of salicylate dose. J Pediatr 1997;131: 888-893.

56. Newburger JW, Takahashi M, Beiser AS, et al. A single intravenous infusion of gamma globulin as compared with four infusions in the treatment of acute Kawasaki syndrome. N Engl J Med 1991;324:1633-1639.

57. Sato N, Sugimura T, Akagi T, et al. Selective high dose gammaglobulin treatment in Kawasaki disease: assessment of clinical aspects and cost effectiveness. Pediatr Int 1999;41:1-7.

58. Muta $\mathrm{H}$, Ishii $\mathrm{M}$, Egami $\mathrm{K}$, et al. Early intravenous gamma globulin treatment for Kawasaki disease: the nationwide survey in Japan. J Pediatr 2004;144:496-499.

59. Harada K. Intravenous $\gamma$-globulin treatment in Kawasaki disease. Acta Paediatr Jpn 1991;33:805-810.

60. Wright DA, Newburger JW, Baker A, Sundel RP. Treatment of immune globulin-resistant Kawasaki disease with pulsed doses of corticosteroids. J Pediatr 1996;128:146-149.

61. Kato H, Koike S, Yokoyama T. Kawasaki disease: effect of treatment on coronary artery involvement. Pediatrics 1979;63: $175-179$.

62. Akagi $\mathrm{T}$, Kato $\mathrm{H}$, Inoue $\mathrm{O}$, Sato $\mathrm{N}$. Salicylate treatment in Kawasaki disease: high dose or low dose? Eur J Pediatr 1991, 150:642-646.

63. Yokoyama $T$, Ichinose E, Kato H. Aspirin treatment and platelet function in Kawasaki disease. Kurume Med J 1980;27: 57-60.

64. Suzuki N, Seguchi M, Kouno C, Inukai K, Kato H, Kobayashi $\mathrm{H}$. Rupture of coronary aneurysm in Kawasaki disease. Pediatr Int 1999;41:318-320.

65. Sundel RP, Baker AL, Fulton DR, Newburger JW. Corticosteroids in the initial treatment of Kawasaki disease: report of a randomized trial. J Pediatr 2003;142:611-616.

66. Furukawa S, Matsubara T, Umezawa Y, Motohashi T, Ino T, Yabuta K. Pentoxifylline and intravenous gamma globulin combination therapy for acute Kawasaki disease. Eur J Pediatr 1994;153:663-667.

67. Zaitsu M, Hamasaki $\mathrm{Y}$, Tashiro $\mathrm{K}$, et al. Ulinastatin, an elastase inhibitor, inhibits the increased mRNA expression of prostaglandin $\mathrm{H} 2$ synthase-type 2 in Kawasaki disease. J Infect Dis 2000;181:1101-1109. 
68. Imanaga $T$, Mori $M$, Miyamae $T$, et al. Plasma exchange for refractory Kawasaki disease. Eur J Pediatr 2004;163:263-264.

69. Weiss JE, Eberhard BA, Chowdhury D, Gottlieb BS. Infliximab as a novel therapy for refractory Kawasaki disease. J Rheumatol 2004;31:808-810.

70. Wallace CA, French JW, Kahn SJ, Sherry DD. Initial intravenous gamma globulin treatment failure in Kawasaki disease. Pediatrics 2000;24:145-148.

71. Williams RV, Wilke VM, Tani LY, Minch LL. Does abciximab enhance regression of coronary aneurysms resulting from Kawasaki disease? Pediatrics 2002;109:E4.

72. Ino $\mathrm{T}$, Akimoto $\mathrm{K}$, Ohkubo $\mathrm{M}$, et al. Application of percutaneous transluminal coronary angioplasty to coronary arterial stenosis in Kawasaki disease. Circulation 1996;93:1709-1715.

73. Sugimura T, Yokoi $H$, Sato N, et al. Interventional treatment for children with severe coronary artery stenosis with calcification after long-term Kawasaki disease. Circulation 1997;96: 3928-3933.

74. Ishii $M$, Ueno $T$, Akagi $T$, et al. Guidelines for catheter intervention in coronary artery lesion in Kawasaki disease. Pediatr Int. 2001;43:558-562.

75. Kato $\mathrm{H}$, Ishii $\mathrm{M}$, Akagi $\mathrm{T}$, et al. Interventional catheterization in Kawasaki disease. J Intervent Cardiol 1998;11:355-361.

76. Akagi T, Ogawa S, Ino T, et al. Catheter interventional treatment in Kawasaki disease: a report from the Japanese Pediatric Interventional Cardiology Investigation group. J Pediatr. 2000; 137:181-186.

77. Ishii $M$, Ueno $T$, Ikeda $H$, et al. Sequential follow-up results of catheter intervention for coronary artery lesions after Kawasaki disease: quantitative coronary artery angiography and intravascular ultrasound imaging study. Circulation 2002; 105:3004-3010.

78. Kitamura S, Kameda Y, Seki T, et al. Long-term outcome of myocardial revascularization in patients with Kawasaki coronary artery disease: a multicenter cooperative study. J Thorac Cardiovasc Surg 1994;107:663-673.

79. Tsuda E, Kitamura S. Cooperative study group of Japan: National survey of coronary artery bypass grafting for coronary stenosis caused by Kawasaki disease in Japan. Circulation 2004;110/suppl 1):II 61-66.

80. Guideline for treatment and management of cardiovascular sequelae in Kawasaki disease. Subcommittee of Cardiovascular Sequelae, Subcommittee of Surgical Treatment, Kawasaki Disease Research Committee. Heart Vessels 1987;3:50-54.

81. Yoshikawa Y, Yagihara T, Kameda Y, et al. Result of surgical treatments in patients with coronary-arterial obstructive disease after Kawasaki disease. Eur J Cardiothorac Surg 2000;17: 515-519.

82. Checchia PA, Pahl E, Shaddy RE, Shulman ST. Cardiac transplantation for Kawasaki disease. Pediatrics 1997;100:695-699.

83. Kato H. Long-term consequences of Kawasaki disease: pediatrics to adults. In: Kato $\mathrm{H}$, ed. Kawasaki Disease. Amsterdam: Elsevier, 1996:557-566.

84. Ichinose E, Inoue O, Hiyoshi $\mathrm{Y}$, Kato H. Fate of coronary aneurysms in Kawasaki disease: analysis of prognostic factors. In: Doyle EF, Engle MA, Gersony W, eds. Springer: New York, Pediatric Cardiology. 1986:1099-1101.
85. Sasaguri Y, Kato H. Regression of aneurysms in Kawasaki disease: a pathologic study. J Pediatr 1982;100:225-231.

86. Hayakawa S, Nakamura Y, Yashiro M, et al. Analyses of fatal cases of Kawasaki disease in Japan using vital statistical data over 27 years. J Epidemiol 2003;13:246-250.

87. Nakamura Y, Yanagawa H, Harada $\mathrm{K}$, et al. Mortality among persons with a history of Kawasaki disease in Japan. The fifth look. Arch Pediatr Adolesc Med 2002;156:162-165

88. Suzuki A, Miyagawa-Tomita S, Komatsu K, et al. Active remodeling of the coronary arterial lesions in the late phase of Kawasaki disease: immunohistochemical study. Circulation 2000;101:2935-2941.

89. Suzuki A, Miyagawa-Tomita S, Komatsu K, et al. Immunohistochemical study of apparently intact coronary artery in a child after Kawasaki disease. Pediatr Int 2004;46:590-596.

90. Sugimura T, Kato H, Inoue O, Takagi J, Fukuda T, Sato N. Vasodilatory response of the coronary arteries after Kawasaki disease: evaluation by intracoronary injection of isosorbide dinitrate. J Pediatr 1992;121:684-688.

91. Yamakawa R, Ishii M, Sugimura T, et al. Coronary endothelial dysfunction after Kawasaki disease: evaluation by intracoronary injection of acetylcholine. J Am Coll Cardiol 1998;31: 1074-1080

92. Iemura $M$, Ishii $M$, Sugimura T, Akagi T, Kato H. Long term consequences of regressed coronary aneurysms after Kawasaki disease: vascular wall morphology and function. Heart 2000 83:307-311.

93. Dhillon R, Clarkson P, Donald AE, et al. Endothelial dysfunction late after Kawasaki disease. Circulation 1996;94:21032106.

94. Mitani Y, Okuda Y, Shimpo H, et al. Impaired endothelial function in epicardial coronary arteries after Kawasaki disease. Circulation 1997;96:454-461.

95. Silva AA, Maeno Y, Hashmi A, Smallhorn JF, Silverman ED, McCrindle BW. Cardiovascular risk factors after Kawasaki disease: a case-control study. J Pediatr 2001;138:400-405.

96. Mitani Y, Sawada H, Hayakawa H, et al. Elevated levels of highsensitivity C-reactive protein and serum amyloid-A late after Kawasaki disease: association between inflammation and late coronary sequelae in Kawasaki disease. Circulation 2005;111: $38-43$.

97. Takahashi K, Oharaseki T, Naoe S. Pathological study of postcoronary arteritis in adolescents and young adults: with reference to the relationship between sequelae of Kawasaki disease and atherosclerosis. Pediatr Cardiol. 2001;22:138-142.

98. Kato $\mathrm{H}$, Inoue $\mathrm{O}$, Kawasaki $\mathrm{T}$, Fujiwara $\mathrm{H}$, Watanabe $\mathrm{T}$, Toshima $\mathrm{H}$. Adult coronary artery disease probable due to childhood Kawasaki disease. Lancet 1992;340:1127-1129.

99. Burns JC, Shike H, Gordon JB, Malhotra A, Schoenwetter M, Kawasaki T. Sequelae of Kawasaki disease in adolescents and young adults. J Am Coll Cardiol 1996;28:253-257.

100. American Heart Association. Guidelines for diagnosis and treatment of Kawasaki disease. Circulation 1993;87:17761780

101. Guidelines for diagnosis and management of cardiovascular sequelae in Kawasaki disease. Japanese Circulation Society. Circ J 2003;67(suppl IV):1111-1173 (in Japanese). 